\title{
Endodontics and COVID-19 -
}

\section{where are we now?}

\author{
Ruvimbo Mukonoweshuro, \\ Kathryn Mulhearn and \\ Manal Ablal
}

\section{Introduction}

The impact of COVID-19 on dentistry has been profound with clinicians at high risk of exposure to the virus. At the beginning of the pandemic, many endodontic emergencies presented to an urgent dental care centre for treatment with a considerable amount of affected teeth resulting in extraction. Now two years on from the start of the pandemic, primary care services have remobilised and many of the adapted protocols introduced for emergency endodontic care remain in place despite the introduction of vaccines and easing of public restrictions.

Since the reopening of primary care dentistry in June 2020 there has been a back-log of patients who are keen to save teeth and who require endodontic care to stabilise disease. With new variants arising and the ongoing easing and reimplementation of restrictions in the UK, it remains imperative to mitigate the risk of disease transmission when undertaking endodontic treatment. This article aims to review the current guidance in light of the most recent British Endodontic Society (BES) guidelines released on 1 December 2021 to ensure safe endodontic treatment in general practice in the presence of COVID-19 and its new variant, Omicron.

\section{Current guidelines}

\section{Triage}

It remains essential that patients presenting for endodontic care are screened via telephone prior to arranging an appointment, including carrying out risk assessments prior to consultation to identify vulnerable people at risk of contracting COVID-19. ${ }^{1}$

During the height of the pandemic, some practices implemented remote triaging/teledentistry which proved successful for many. ${ }^{2}$ Therefore, where facilities exist, current guidance states that it is advisable to continue with this method for remote consultation. ${ }^{1}$ For those patients identified as vulnerable, it is advisable for them to be seen as the first appointment of the day. ${ }^{1}$

\section{In practice}

On arrival for the appointment, it is a matter of professional judgement whether the patient's temperature is taken, however, hand hygiene and reconfirming COVID-19 status remain essential, along with social distancing measures in waiting rooms. ${ }^{1}$ Masks are mandatory for all patients upon arrival into the dental practice and electronic payments are recommended and advised. ${ }^{1}$

\section{Endodontic procedure}

There is some conflicting advice as to whether a pre-operative mouth rinse should be used before commencing endodontic procedures. ${ }^{1}$ Recent research identified that cetylpyridinium chloride and povidone-iodine mouthwashes are effective in reducing COVID-19 salivary load. ${ }^{3}$ Similarly, another study found that povidoneiodine mouthwashes effectively reduce COVID19 infectivity. ${ }^{4,5}$ Conversely, a differing study stated that the use of hydrogen peroxide and chlorhexidine -containing mouthwashes are ineffective in reducing COVID-19 intraorally. ${ }^{4}$ Despite the conflicting evidence the current guidance advises the use of pre-operative mouth rinse for at least 30 seconds prior to commencing endodontic treatment, specifically recommended is a $1 \%-1.5 \%$ hydrogen peroxide or $0.2 \%$ povidone-iodine rinse for one minute. ${ }^{1,6}$

Recent guidelines recommend single visit endodontic treatment where possible to reduce the number of visits. ${ }^{1}$ This can be achieved by core placement or indirect restoration under dental dam at the time of obturation.

\section{Radiography}

During the height of the pandemic extra-oral radiographs such as CBCT and OPG were recommended to reduce viral contamination risk, however, diagnostic Intra-oral radiographs may now be utilised again with adherence to universal precautions such as disinfecting film packets and surfaces
Key points

$\rightarrow$ Enhanced PPE, adapted treatment protocols and fallow times remain key clinical challenges with regard to emergency endodontic care

$\rightarrow$ Dental professionals must continue to ensure patient and colleague safety with the introduction of COVID-19 variants

$\rightarrow$ A clear roadmap and official guidance is required to direct the ongoing management of acute endodontic emergencies

including control panels and ensuring clean and dirty zones for films and film holders. ${ }^{7}$

\section{Cross-infection control}

For dental clinical staff, adequate and thorough disinfection of the clinical area is required alongside constant change of keyboard barriers between patients. For aerosol generating procedures (AGP), donning an enhanced level PPE consisting of FFP3/N95 mask/PAPR, visor, gown, hat plus shoe covers are vital in view of the risk of viral contamination. Whereas non AGPs and general patient contact require a type IIR mask, apron and visor. ${ }^{1}$ For endodontic procedures requiring the use of microscopes, modified PPE such as a fullface visor attached to the dental microscope can be utilised to reduce risk of COVID-19 transmission and contamination. ${ }^{8}$

Similar to previous guidelines, dental dam remains a gold standard aspect to endodontic treatment ${ }^{1}$. Single tooth isolation with use of caulking agents such as Opaldam or Oraseal are recommended to maximise oral sterility. ${ }^{9}$ In order to achieve greater oral sterility, guidelines currently advise the use of $3 \%$ sodium hypochlorite or $1.5 \%$ hydrogen peroxide for operative field decontamination which includes the isolated tooth and dental dam ${ }^{1}$. Two recent studies concluded that dental dam isolation successfully reduces bioaerosols in the dental clinical environment. ${ }^{10,11}$ This evidence supports the current guidelines which advocate the use of dental dam to reduce the transmission risk of COVID-19. 
High volume aspiration remains essential for AGPs. ${ }^{12}$ Several studies investigated the efficacy of several suction devices and found that the use of high-volume suction significantly reduces bioaerosols that are close to the simulated oral cavity. ${ }^{13,14}$ Additional research states that highvolume suction, alongside an air cleaning system are effective in reducing aerosol particles. ${ }^{15}$ Although the certainty of the evidence is low due to the fact the studies were conducted using simulated dental procedures on dental manikins, the latest guidelines encourage the use of high-volume suction to reduce the potential risk of COVID-19 transmission. ${ }^{9}$

Whilst biomechanical preparation including instrumentation of the canals is now appropriate during endodontic treatment, refinement of the access cavity should be completed with a slow speed with no or minimal water to minimise aerosol generation. Additionally, 3-in-1 use should be avoided and any irrigation must be carried out with a syringe.

Recent recommendations state that where possible, it is advisable to commence endodontic treatment in a single visit to reduce the number of patient visits. Additionally, AGP procedures including access cavities should be kept to the start of the procedure to minimise fallow time. ${ }^{1}$ A recent study observed the splatter on dental personnel during AGPs and found that there was significant splatter on both the dentist and dental assistant following AGPs, even with the use of high-volume aspiration. ${ }^{16}$ This study confirms the need for mitigation on AGPs to minimise transmission of COVID-19 via aerosol.

After treatment has been completed clinical staff are required to doff their enhanced PPE. ${ }^{1}$ Additionally, hand hygiene remains essential after completing treatment with each patient. ${ }^{1}$

Current evidence demonstrates that a fallow time is appropriate after performing AGPs to minimise the risk of COVID-19 transmission in dental practices. ${ }^{1}$ One study found that aerosol particles are reduced to baseline levels over a short period of time of approximately five minutes in a room with six air changes per hour, ${ }^{17}$ whereas other research stated that a fallow time of around 30 minutes is adequate after an AGP. ${ }^{15}$ Two recent studies suggest that fallow time can be reduced if sufficient highvolume suction and mechanical ventilation of six air changes per hour are utilised. ${ }^{13,15}$ Additionally, further evidence suggested most aerosol settles within 10 minutes after which time decontamination can take place..$^{18}$ It is evident from all the research that a fallow time offers benefit in reducing contaminated aerosol particles, however, there is clearly conflicting evidence regarding the duration of fallow times. Therefore, fallow time duration may be subject to further review in the near future. Overall, the latest guidelines recommend either a 20-minute fallow time or 15 minutes for AGPs that have a duration of five minutes or under to reduce the risk of COVID-19 transmission. ${ }^{1}$

Following the completion of the fallow period, the dental nurse should disinfect the dental surgery in level 2 PPE. ${ }^{1}$ Additionally, it is currently essential that the dental nurse prepares the surgery according to HTM 01-05 and Infection Prevention and Control (IPC) decontamination guidelines. ${ }^{1}$ While the surgery is being disinfected, the dentist must write clinical notes in a different area. ${ }^{1}$

\section{A look into the future}

Many of the changes introduced at the beginning of the pandemic remain in place with advice to continue to take enhanced safety measures for endodontic treatments such as telephone triaging, enhanced PPE, disinfection of the tooth and dental dam, limitation of AGPs where possible, along with single visit treatments and post-procedural fallow times, and with the emergence of the COVID-19 variant Omicron these measures could be set to remain.

A recent Healthwatch England survey revealed that more than half of high street dentists feel Omicron has had a high impact on their willingness to ease restrictions and many are reluctant to relax COVID precautions in practice. ${ }^{19}$ An updated standard operating procedure released by the BES aligns with UK Infection Prevention Control guidance agreed by four Chief Dental Officers and offers a helpful guide for dental practitioners to continue to deliver safe endodontic care. At the time of writing, more than 46.8 million people in the UK have received both doses of a COVID-19 vaccine and over 24 million have received a booster or third vaccine. Due to the newly arising variants of the COVID-19 virus, the NHS are preparing to implement an annual COVID-19 booster vaccination programme.

It appears COVID-19 and its variants may be viruses that have a permanent presence and therefore these current COVID-19 guidelines may become the gold standard practice for endodontic procedures to ensure safety of patients and dental staff. •

\section{References}

1. British Endodontic Society. BES COVID-19 return to work SOP (2021). Available at: https:// britishendodonticsociety.org.uk/wp-content/ uploads/2021/12/BES_SOP_1.5.pdf (Accessed December 2021)
2. Byrne E, Watkinson S. Patient and clinician satisfaction with video consultations during the COVID-19 pandemic: an opportunity for a new way of working. J Orthod 2021; 48: 64-73.

3. Seneviratne C J, Balan P, Ko Kwan Ki K, et al. Efficacy of commercial mouth-rinses on SARS-CoV-2 viral load in saliva: randomized Control Trial in Singapore. Infection 2021; 49: 305-311.

4. Davies K, Buczkowski H, Welch S R, et al. Effective in-vitro inactivation of SARS-CoV-2 by commercially available mouthwashes. J Gen Virol 2021; 102: 001578.

5. Hassandarvish P, Tiong V, Mohamed N A, et al. In vitro virucidal activity of povidone iodine gargle and mouthwash against SARS-CoV-2: implications for dental practice. Br Dent J 2020; doi.org/10.1038/ s41415-020-2402-0.

6. Testori T, Wang H, Basso M et al. COVID-19 and Oral Surgery: A narrative review of preoperative mouth rinses. Acta Stomatologica Croatica 2021; 54: 431-441.

7. Nixon P, Rout J, Brown J, Thomas B. Recommendations for diagnostic imaging during COVID-19 pandemic. 1st ed. British Society of Dental and Maxillofacial Radiology and The Faculty of Dental Surgery, Royal College of Surgeons of England. (2020). p.2-3.

8. Ayub K, Alani A. Acute endodontic and dental trauma provision during the COVID-19 crisis. Br Dent J 2020; 229: 169-175

9. Scottish Dental Clinical Effectiveness Programme. Mitigation of Aerosol Generating Procedures in Dentistry. A Rapid Review (2021). Available from: www. sdcep.org.uk/wp-content/uploads/2021/04/SDCEPMitigation-of-AGPs-in-Dentistry-Rapid-Review-v1.2April-2021.pdf (Accessed November 2021).

10. Samaranayake L P, Fakhruddin K S, Buranawat B, Panduwawala $C$. The efficacy of bio-aerosol reducing procedures used in dentistry: a systematic review. Acta Odontologica Scandinavica 2021; 79: 69-80.

11. Balanta-Melo J, Gutierrez A, Sinisterra G, et al. Rubber Dam Isolation and High-Volume Suction Reduce Ultrafine Dental Aerosol Particles: An Experiment in a Simulated Patient. Applied Sci 2020; 10: 6345-6345.

12. Scottish Dental Clinical Effectiveness Programme. Mitigation of Aerosol Generating Procedures in Dentistry. Report on Recent Published Evidence (2021). Available from: https://www.sdcep.org.uk/wp-content/ uploads/2021/04/SDCEP-Report-on-Recent-PublishedEvidence-April-2021.pdf (Accessed November 2021).

13. Shahdad S, Patel T, Hindocha A, et al. The efficacy of an extraoral scavenging device on reduction of splatter contamination during dental aerosol generating procedures: an exploratory study. Br Dent J 2020; doi. org/10.1038/s41415-020-2112-7.

14. Matys J, Grzech-Lesniak K. Dental Aerosol as a Hazard Risk for Dental Workers. Materials 2020; 13.

15. Ehtezazi T, Evans D G, Jenkinson I D, et al. SARS CoV-2: characterisation and mitigation of risks associated with aerosol generating procedures in dental practices. Br Dent J 2021; doi.org/10.1038/ s41415-020-2504-8.

16. Chanpong B, Tang M, Rosenczweig A, Lok P, Tang R. Aerosol-Generating Procedures and Simulated Cough in Dental Anesthesia. Anesthesia Progress 2020; 67: 127-134.

17. Din A R, Hindocha A, Patel T, et al. Quantitative analysis of particulate matter release during orthodontic procedures: a pilot study. Br Dent J 2020; doi.org/10.1038/s41415-020-2280-5.

18. Holliday R, Allison J R, Currie C C, et al. Evaluating contaminated dental aerosol and splatter in an open plan clinic environment: Implications for the COVID19 pandemic. J Dent 2021; 105: 103565.

19. The Dentist. Omicron threat leaves dentists struggling to restore service. 2021 Available from: www.the-dentist. co.uk/content/news/omicron-threat-leaves-dentistsstruggling-to-restore-service (Accessed December 2021).

https://doi.org/10.1038/s41404-022-1027-1 